\title{
Síndrome de burnout en enfermeros de hospitales públicos y privados en Guayaquil, Ecuador
}

\author{
Burnout syndrome in nursing staff of public and private hospitals in \\ Guayaquil, Ecuador
}

Síndrome de Burnout em enfermeiras de hospitais públicos e privados de Guayaquil, Equador

a Departamento de Enfermería, Servicio de Cirugía Cardiovascular, Hospital Clínica Guayaquil, Guayaquil, Ecuador.

\section{Correspondencia a:}

Eliana Marilin Cerón López, paola.elmarice02@gmail.com

Recibido: 19 de octubre, 2020 Aceptado: 17 de noviembre, 2020 Publicado: 30 de diciembre, 2020

\section{ARTÍCULO ORIGINAL}

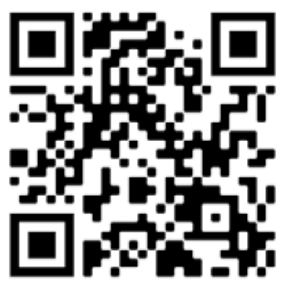

Escanea en tu dispositivo móvil o revisa este artículo en: https:// revistaclinicaguayaquil.org

\author{
Eliana Marilin Cerón López (D) a
}

\begin{abstract}
RESUMEN
Introducción: El síndrome de burnout, también llamado síndrome del trabajador quemado, constituye una respuesta al estrés laboral crónico. Los profesionales que tienen interacción directa y constante con otras personas, constituyen el grupo de mayor riesgo. Es así que los profesionales de enfermería, se consideran uno de los grupos más vulnerables para desarrollarlo.
\end{abstract}

Materiales y Métodos: Se diagnosticó e identificó la prevalencia del síndrome de burnout y las variables asociadas al mismo, también se analizaron sus tres dimensiones: agotamiento emocional, despersonalización y realización personal en enfermeros de hospitales y clínicas privadas y públicas de la ciudad de Guayaquil. Este es un estudio de tipo descriptivo de corte transversal cuantitativo y correlacional. Se aplicó la escala de Maslach Burnout Inventory validada al español y se correlacionó con datos sociodemográficos y laborales reportados por los enfermeros participantes del estudio. Resultados: La prevalencia del síndrome de burnout fue del $1.7 \%$. El $6.6 \%$ y el $5.2 \%$ presentaron nivel alto de agotamiento emocional y despersonalización, respectivamente; el $14.6 \%$ presentó un nivel bajo en realización personal. Se encontró asociación significativa entre la variable sector laboral (público, privado o mixto) y el síndrome de burnout en sus tres dimensiones ( $p$ 0.01).

Conclusiones: Si bien no se evidenciaron niveles críticos del síndrome de burnout, se observó niveles altos de desgaste emocional en profesionales que trabajan en el sector público, siendo más frecuente en el sexo masculino.

Palabras clave: síndrome de burnout; estrés; variables organizacionales

\section{ABSTRACT}

Introduction: The burnout syndrome, also called burnout worker syndrome, constitutes a response to chronic work stress. Professionals who have direct and constant interaction with other people are the highest risk group. Thus, nursing professionals are considered one of the most vulnerable groups to develop it.

Materials and Methods: The prevalence of burnout syndrome and the variables associated with it were identified; its three dimensions (emotional exhaustion, depersonalization and personal fulfillment) were also analyzed in nurses from private and public hospitals and clinics in the city of Guayaquil. This is a descriptive, quantitative and correlational cross-sectional study. The Maslach Burnout Inventory scale validated in spanish was applied, and it was correlated with sociodemographic and work data reported by the nurses participating in the study. 
Results: The prevalence of the burnout syndrome was $1.7 \%$. Six and six tenths (6.6) percent and $5.2 \%$ had a high level of emotional exhaustion and depersonalization, respectively; $14.6 \%$ of the population presented a low level of personal fulfillment. A significant association was found between the variable of the working sector (public, private or mixed) and the burnout syndrome in its three dimensions ( $p$ 0.01).

Conclusions: Although critical levels of burnout syndrome were not evidenced, high levels of emotional exhaustion were observed in professionals working in the public sector, being more frequent in males.

Key words: burnout syndrome; stress; organizational variables

\section{RESUMO}

Introdução: A síndroma de burnout, também chamado de síndroma do trabalhador de burnout, é uma resposta ao estresse laboral crônico. Profissionais que têm interação direta e constante com outras pessoas são o grupo de maior risco. Assim, os profissionais de enfermagem são considerados um dos grupos mais vulneráveis para desenvolvê-lo.

Materiais e Métodos: Foi diagnosticada e identificada a prevalência do Síndroma de Burnout junto as variáveis a ela associadas, assim foram analisadas suas três dimensões: exaustão emocional, despersonalização e realização pessoal em enfermeiras de hospitais públicos e particulares da cidade de Guayaquil. Trata-se de um estudo transversal descritivo, quantitativo e correlacional. Foi aplicada a escala Maslach Burnout Inventory validada em língua espanhola e correlacionada com dados sociodemográficos de trabalhadores da área da saúde, ou seja, enfermeiros participantes do estudo.

Resultados: A prevalência do síndroma de burnout foi de 1,7\%. $6,6 \%$ e $5,2 \%$ apresentaram alto nível de exaustão emocional e despersonalização, respectivamente; 14,6\% apresentaram baixo nível de realização pessoal. Foi encontrada associação significativa entre a variável setor de trabalho (público, particular ou misto) e a síndroma de burnout em suas três dimensões ( $p$ 0,01).

Conclusões: Apesar de não haver níveis críticos de síndroma de burnout, foram observados elevados níveis de exaustão emocional nos profissionais que atuam no setor público, sendo mais frequente no gênero masculino.

Palavras-chave: síndroma de burnout; estresse; variáveis organizacionais

\section{INTRODUCCIÓN}

El síndrome de burnout, conocido también como síndrome de desgaste ocupacional o del trabajador quemado, es un padecimiento que se desencadena como respuesta a presiones prolongadas que una persona sufre ante factores estresantes emocionales relacionados con el trabajo (1).

El ámbito laboral, en cualquiera de sus presentaciones o empleos, genera un nivel de estrés creado por las múltiples actividades y exigencias de cada uno de los puestos de trabajo. Se ha demostrado que en el campo de la salud este estrés es mucho mayor, ya que el trabajar con personas de forma directa resulta más demandante, debido a la interacción constante durante largas horas de trabajo. Los profesionales sanitarios, en este caso, los licenciados, técnicos y auxiliares de enfermería, constituyen un grupo de trabajadores que se haya en continuo contacto con personas que tienen diferentes patologías y motivos de sufrimiento (2). La forma de actuar y afrontar estas situaciones afecta directamente el estado físico y mental de los trabajadores de la salud; el agotamiento, así como la sensación de ineficacia en el trabajo y despersonalización, puede afectar el desempeño de sus actividades laborales.

Tanto el estrés laboral, como el síndrome de burnout, son situaciones a las que el ser humano se enfrenta en sus horas laborables, pero sin embargo, no se refieren a lo mismo. El término estrés describe procesos temporales breves, por otro lado, el síndrome de burnout se refiere a una respuesta al estrés laboral crónico que se manifiesta con una serie de actitudes negativas hacia el propio trabajo, la organización, y el desarrollo personal (3). El burnout representa una situación de agotamiento emocional, cada vez más frecuente entre los profesionales que prestan sus servicios a través de una relación directa con personas que padecen alguna enfermedad, así como también con los familiares de los mismos (2).

En 1974, el psiquiatra Herbert Freudenberg, fue el primero en documentar en la literatura científica la palabra: burnout ("estar quemado"), con un significado similar al que le aplicamos en el contexto actual (4). No obstante, este término ya se utilizaba en el mundo del arte, o del deporte. Freudenberg, trabajaba como voluntario en una clínica de Nueva York, con pacientes toxicómanos y de hecho, en un primer momento, usaba esta palabra para referirse a los efectos del consumo crónico de sustancias tóxicas (4). Constató como sus propios compañeros, tras un periodo de tiempo relativo, experimentaban un "patrón conductual homogéneo" caracterizado por agotamiento, pérdida de motivación y una serie de síntomas asociados compatibles con la ansiedad y la depresión $(4,5)$. 
Así también, la psicóloga social Cristina Maslach, estudió en el año 1981 las reacciones que experimentaban las personas que trabajaban dentro de las profesiones de servicio-ayuda, caracterizadas por una relación continua de ayuda hacia el paciente, turnos rotatorios, muchas horas de trabajo, sueldos escasos, elevada responsabilidad y tensión $(4,6)$. Junto con Susan E. Jackson, definen el burnout como un síndrome de agotamiento emocional, despersonalización y falta de realización personal en el trabajo que puede desarrollarse como consecuencia de la exposición continua a estresores laborales (6).

En este sentido, enfermería se ha catalogado como el grupo de profesionales de mayor riesgo para adquirir este síndrome de burnout, debido a múltiples factores personales, profesionales e institucionales que desencadenan una serie de respuestas negativas o positivas. Además, se considera que el área de trabajo es un factor importante en el desarrollo del síndrome de burnout en el personal de enfermería, ya que se ha descrito que áreas como emergencias y las unidades de cuidados intensivos son los lugares donde se genera mayor estrés laboral, debido a las altas exigencias que estas representan para la atención de pacientes (7).

\section{MATERIALES Y MÉTODOS}

Estudio de tipo descriptivo de corte transversal, para el cual la muestra de estudio se obtuvo de 219 licenciados, técnicos y auxiliares de enfermería que trabajan en hospitales y clínicas públicos y/o privados de la ciudad de Guayaquil, Ecuador. La recolección de información se realizó mediante un cuestionario digital realizado en Google Forms el cual fue diligenciado voluntariamente, previo a consentimiento informado verbal de cada uno de los participantes. Los datos fueron tabulados en una base de Google Sheets.

El instrumento utilizado fue el cuestionario Maslach Burnout Inventory en su versión en español, adaptada a los profesionales de la salud. Esta escala consiguió una alta consistencia interna y una fiabilidad cercana al 90\%; estuvo constituido por veintidós 22 ítems en forma de afirmaciones, sobre los sentimientos $y$ actitudes del profesional en su trabajo y hacia los pacientes; su función es medir el desgaste profesional (8).

Los veintidós ítems estuvieron subdivididos en los tres aspectos del síndrome de burnout el primero relacionado con la Subescala de agotamiento emocional. El constó de 9 preguntas, valora la vivencia de estar exhausto emocionalmente por las demandas del trabajo; su puntuación máxima es 54 puntos; segundo se tiene la subescala de despersonalización. Está formada por cinco ítems y valora el grado en que cada uno reconoce actitudes de frialdad y distanciamiento; la puntuación máxima es de 30 puntos. Por último, se usó la subescala de realización personal. Se dispuso de ocho ítems. Este evalúa los sentimientos de autoeficacia y realización personal en el trabajo; su puntuación máxima es de 48 puntos.

Para identificar el síndrome de burnout se realiza la suma de las tres dimensiones de la encuesta y, las puntuaciones altas en cansancio emocional y despersonalización y bajas en realización personal definen el síndrome de burnout. Además cada una de la subescalas tiene una puntuación según se describe a continuación, (Tabla 1).

Tabla 1. Dimensiones Cuestionario Burnout

\begin{tabular}{lccc}
\hline & Bajo & Medio & Alto \\
\hline $\begin{array}{l}\text { Cansancio } \\
\text { emocional }\end{array}$ & $0-18$ & $19-26$ & $27-54$ \\
Despersonalización & $0-5$ & $6-9$ & $10-30$ \\
Realización personal & $0-33$ & $34-39$ & $40-56$
\end{tabular}

Tomado de: Ecestaticos. Cuestionario Burnout - Maslach Burnout Inventory [Internet]. [Citado el 2020 Oct 1]. Disponible en: https://www.ecestaticos.com/file/035b15 f97effea4c69fd6519e8114bcf/1515665476-cuestionario_ burnout.pdf

Para el análisis estadístico los datos fueron exportados a un libro de Microsoft Excel y posteriormente se utilizó el software XLSTAT versión 2020.4.1.1030. Se hizo un análisis descriptivo de todas las variables. En el caso de las variables cualitativas se calcularon frecuencias absolutas y porcentajes; para las variables cuantitativas se incluyeron medidas de tendencia central como media y desviación estándar; para aquellas variables con distribución no normal se utilizó mediana y rango intercuartil. Se realizó el análisis correlacional entre las variables sociodemográficas con cada una de las dimensiones del síndrome, y se calificó como estadísticamente significativas aquellas que obtuvieron un valor de $p<0.05$.

\section{RESULTADOS}

Se recolectaron 219 respuestas al cuestionario, de las cuales 4 de ellas fueron eliminadas por información incompleta. En cuanto a las características sociodemográficas de los enfermeros que trabajan en las diferentes 
clínicas y hospitales de Guayaquil, se obtuvo que, de los 215 encuestados, el sexo femenino correspondió a un $77.2 \%(n=166)$ y el sexo masculino con un $22.8 \%(n=49)$. El $44.2 \%(n=95)$ corresponde al grupo etario comprendido entre 20 y 30 años, y, el sector privado representó el mayor número de encuestados en comparación al sector público, y una muestra menor de enfermeros que trabajan tanto en lo público como privado, obteniendo así el $79.1 \%(n=170)$, $20.0 \%(n=43)$, y $0.9 \%(n=2)$ respectivamente.

Los enfermeros que diligenciaron la encuesta pertenecen a diferentes áreas clínicas y quirúrgicas, el grupo con mayor representación fue el del área de hospitalización con un 43.7\% ( $n=94)$, seguido de unidad de cuidados intensivos con un $24.2 \%(n=52)$ y el sector de emergencias con el 20.5\% ( $n=44)$. El grupo con menor encuestados correspondió a aquellos que trabajan en áreas administrativas o de docencia, constituyendo el $2.8 \% \quad(n=6)$ de la población. En cuanto a las horas laborales, el 82.3\% de los encuestados trabajan entre 8 a 12 horas al día, y el $55.3 \%$ de la población asiste a la jornada diurna; solo un 6.5\% $(n=14)$ trabajan en más de dos lugares (Tabla 2 ).

Tabla 2. Características sociodemográficas de la población.

\begin{tabular}{|c|c|}
\hline & n (\%) \\
\hline $\mathrm{n}$ & 215 \\
\hline Edad (años)* & $31.0(R I Q) 14.0)$ \\
\hline \multicolumn{2}{|l|}{ Grupos etarios } \\
\hline$<30$ & $95(44.2)$ \\
\hline $30-39$ & $60(27.9)$ \\
\hline $40-49$ & $38(17.7)$ \\
\hline $50-59$ & $17(7.9)$ \\
\hline$\geq 60$ & $5(2.3)$ \\
\hline \multicolumn{2}{|l|}{ Sexo } \\
\hline Femenino & $166(77.2)$ \\
\hline Masculino & $49(22.8)$ \\
\hline \multicolumn{2}{|l|}{ Lugar de trabajo } \\
\hline Público & $43(20.0)$ \\
\hline Privado & $170(79.1)$ \\
\hline Mixto & $2(0.9)$ \\
\hline \multicolumn{2}{|l|}{ Área } \\
\hline Hospitalización & $94(43.7)$ \\
\hline Unidad de Cuidados Intensivos & $52(24.2)$ \\
\hline Emergencias & $44(20.5)$ \\
\hline Quirófano & $10(4.7)$ \\
\hline Consulta externa, atención primaria & $9(4.2)$ \\
\hline Otros & $6(2.8)$ \\
\hline \multicolumn{2}{|l|}{ Horas de trabajo/día } \\
\hline Menos de 8 & $5(2.3)$ \\
\hline De 8 a 12 & $177(82.3)$ \\
\hline Más de 12 & $33(15.3)$ \\
\hline \multicolumn{2}{|l|}{ Jornada laboral } \\
\hline Diurna & $119(55.3)$ \\
\hline Nocturna & $76(35.3)$ \\
\hline N/R & $20(9.3)$ \\
\hline \multicolumn{2}{|l|}{ Trabajo en más de 1 lugar } \\
\hline Si & $14(6.5)$ \\
\hline No & 201 (93.5) \\
\hline
\end{tabular}

* Expresado en mediana (rango intercuartil). RIQ: rango intercuartil. 
En cuanto al análisis por cada una de las dimensiones del síndrome de burnout, se puede evidenciar que la subescala de cansancio emocional y despersonalización se encuentran en nivel bajo con 8.05 y 2.44 puntos, respectivamente. En cambio, la subescala de realización personal se encontró en un nivel alto con 41.75 puntos (Figura 1), según los valores de referencia establecidos para dicha clasificación.

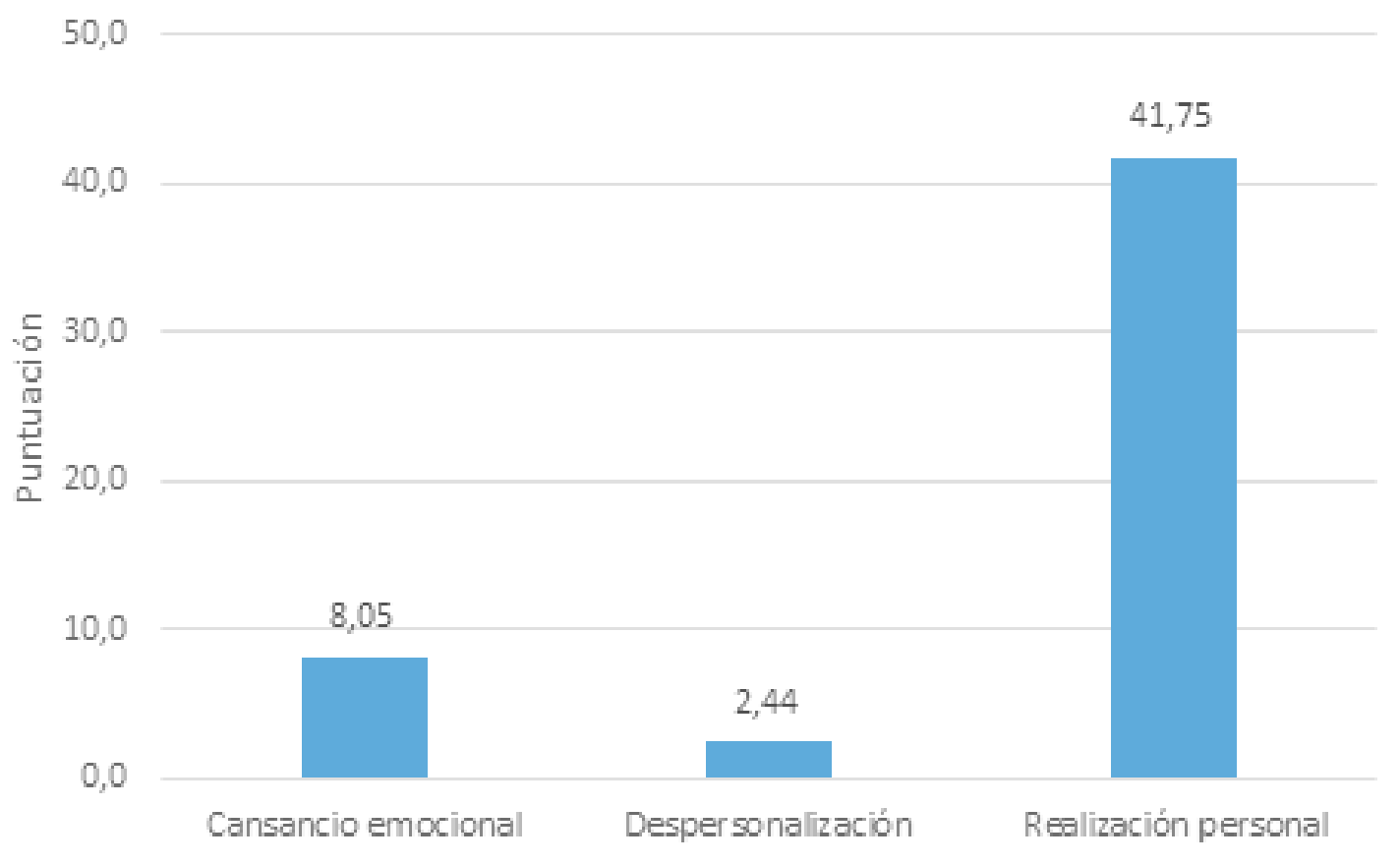

Figura 1. Puntuaciones de las subescalas del síndrome de burnout.

Al desglosar las dimensiones del síndrome de burnout se puede evidenciar que el $85.8 \%(n=181)$ de la población presentó niveles bajos de cansancio emocional, el 7.6\% $(n=16)$ presentó un nivel medio y, el 6.6\% ( $n=14)$ nivel alto (Figura 2). Así también, en la subescala de despersonalización, el 86.7\% $(n=183)$ presentó un nivel bajo, el $8.1 \%(n=17)$ nivel medio, y el 5.2\% $(n=11)$ un nivel alto (figura 3). En la última subescala, acerca de la realización personal, el 14.6\% $(n=31)$ presentó nivel bajo, el 9.4\% ( $n=20)$ nivel medio y un $75.9 \%$ ( $n=161$ ) un nivel alto (Figura 4); estos datos representan que la prevalencia del síndrome de burnout en esta población es baja.

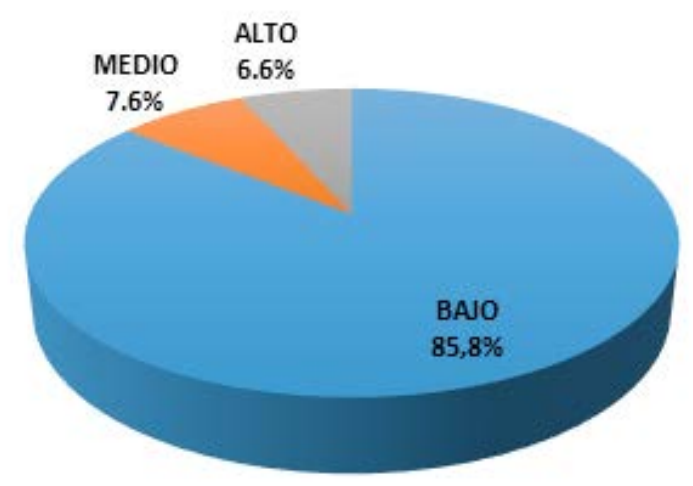

Figura 2. Distribución del cansancio emocional.

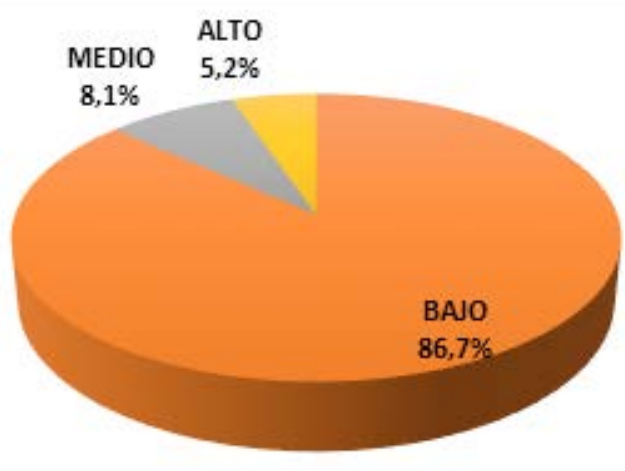

Figura 3. Distribución de la despersonalización. 


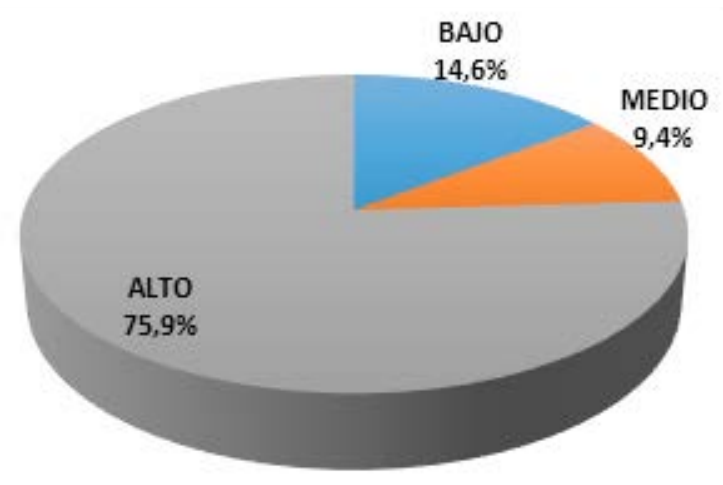

Figura 4. Distribución de la dimensión realización personal.

\section{Asociación de las variables sociodemográficas con las dimensiones del síndrome de burnout}

En cuanto al análisis de las variables sociodemográficas y las dimensiones del síndrome de burnout, aunque el estudio no arrojó valores estadísticamente significativos en las variables, se pudo evidenciar que los hombres presentan mayor cansancio emocional (10.3 \pm 13.0 versus $7.5 \pm 9.9, p 0.40$ ). Aquellos pertenecientes al grupo etario de 30 a 39 años también presentaron un nivel mayor de cansancio emocional $(p=0.07)$. En cambio, si existió significancia estadística en cuanto a las personas que trabajan en el sector público versus al sector privado en cada una de las subescalas: cansancio emocional (16.0 \pm 14.7 versus $6.2 \pm 8.5 ; p<0,0001)$, despersonalización $(4.3 \pm 6.1$ versus $1.9 \pm 3.4 ; p=0.01)$, y realización personal (38.6 \pm 8.8 versus $42.6 \pm 78 ; p=0.01$ ) (Tabla 3 ).

Tabla 3. Asociación de las variables sociodemográficas con las dimensiones del síndrome de burnout.

\begin{tabular}{|c|c|c|c|c|c|c|c|}
\hline \multicolumn{8}{|c|}{ Dimensiones del síndrome de burnout } \\
\hline Variable & Categoría & $\begin{array}{l}\text { Cansancio } \\
\text { emocional }\end{array}$ & $\mathbf{p}$ & Despersonalización & $\mathbf{p}$ & $\begin{array}{c}\text { Realización } \\
\text { personal }\end{array}$ & $\begin{array}{l}\text { Variable } \\
\text { Categoría }\end{array}$ \\
\hline \multirow{2}{*}{ Sexo } & Femenino & $7.5 \pm 9.9$ & \multirow{2}{*}{0.40} & $2.3 \pm 4.2$ & \multirow{2}{*}{0.26} & $41.6 \pm 7.5$ & \multirow{2}{*}{0.74} \\
\hline & Masculino & $10.3 \pm 13.0$ & & $2.8 \pm 4.1$ & & $41.8 \pm 8.4$ & \\
\hline \multirow{4}{*}{ Edad } & $20-29$ & $8.1 \pm 9.7$ & \multirow{4}{*}{0.07} & $2.3 \pm 3.4$ & \multirow{4}{*}{0.55} & $42.0 \pm 7.0$ & \multirow{4}{*}{0.91} \\
\hline & $30-39$ & $9.8 \pm 12.7$ & & $2.7 \pm 4.6$ & & $40.8 \pm 10.1$ & \\
\hline & $40-49$ & $4.6 \pm 8.3$ & & $1.5 \pm 2.7$ & & $42.8 \pm 6.5$ & \\
\hline & 50 o más & $9.8 \pm 12.0$ & & $3.7 \pm 6.8$ & & $41.3 \pm 9.5$ & \\
\hline \multirow{3}{*}{$\begin{array}{l}\text { Sector } \\
\text { de } \\
\text { trabajo }\end{array}$} & Público & $16.0 \pm 14.7$ & \multirow{3}{*}{$<0.0001$} & $4.3 \pm 6.1$ & \multirow{3}{*}{0.01} & $38.6 \pm 8.8$ & \multirow{3}{*}{0.01} \\
\hline & Privado & $6.2 \pm 8.5$ & & $1.9 \pm 3.4$ & & $42.6 \pm 7.8$ & \\
\hline & Mixto & $2.0 \pm 2.8$ & & $1.5 \pm 2.1$ & & $44.5 \pm 3.5$ & \\
\hline \multirow{3}{*}{$\begin{array}{l}\text { Horas de } \\
\text { trabajo }\end{array}$} & Menos de 8 & $6.0 \pm 5.0$ & \multirow{3}{*}{0.99} & $3.2 \pm 3.6$ & \multirow{3}{*}{0.71} & $27.6 \pm 19.3$ & \multirow{3}{*}{0.45} \\
\hline & 8 a 12 & $7.9 \pm 10.3$ & & $2.2 \pm 3.8$ & & $42.0 \pm 7.7$ & \\
\hline & Más de 12 & $9.7 \pm 13.2$ & & $3.2 \pm 5.7$ & & $42.6 \pm 6.1$ & \\
\hline \multirow{2}{*}{$\begin{array}{l}\text { Jornada } \\
\text { laboral }\end{array}$} & Diurna & $9.2 \pm 12.1$ & \multirow{2}{*}{0.33} & $2.7 \pm 4.7$ & \multirow{2}{*}{0.2} & $40.9 \pm 9.0$ & \multirow{2}{*}{0.46} \\
\hline & Nocturna & $6.8 \pm 9.0$ & & $2.0 \pm 3.5$ & & $42.6 \pm 7.0$ & \\
\hline \multirow{2}{*}{$\begin{array}{l}\text { Trabajo } \\
\text { en más } \\
\text { de } 1 \\
\text { lugar }\end{array}$} & $\mathrm{Si}$ & $9.1 \pm 10.5$ & \multirow[b]{2}{*}{0.61} & $2.4 \pm 4.3$ & \multirow[b]{2}{*}{0,44} & $42.3 \pm 8.2$ & \multirow[b]{2}{*}{0.57} \\
\hline & No & $8.0 \pm 10.8$ & & $1.6 \pm 1.2$ & & $41.7 \pm 8.2$ & \\
\hline
\end{tabular}




\section{DISCUSIÓN}

A pesar de que no se evidenciaron niveles críticos del síndrome de burnout, y que los hallazgos de este estudio demostraron que no se alcanzó significancia estadística global, sí se observó niveles altos de desgaste emocional en profesionales que trabajan en el sector público, siendo más frecuente en el sexo masculino. Los resultados indican una baja prevalencia del síndrome de burnout en comparación con otros estudios realizados en otros países como Paraguay; Eulalia Maldonado y Margarita Sacudio, en su estudio evidenciaron una prevalencia del $18.9 \%$ del síndrome de burnout, obteniendo niveles altos en cansancio emocional, nivel medio en despersonalización y un nivel alto en realización personal (3). Sin embargo, existen estudios que comparten resultados similares con el nuestro, como el de Muñoz et al., un estudio realizado en Popayán, Colombia, donde demostraron que la prevalencia del síndrome de burnout fue del 1.7\% (9). El síndrome de burnout tiene importantes repercusiones en el ámbito laboral y personal, sobre todo en los servicios de enfermería, en los cuales el personal se encuentra constantemente expuesto a altas exigencias de trabajo $(2,5,7)$.

Se concluye que este estudio constituye un aporte a la comprensión del síndrome de burnout en los profesionales de enfermería; no obstante, se consideran necesarias más investigaciones que permitan analizar y determinar cómo se comporta el síndrome con referencia a variables sociodemográficas y laborales analizadas y otras no consideradas en este trabajo, $y$, a partir de los hallazgos, establecer estrategias encaminadas a generar cambios organizacionales que fomenten las adecuadas condiciones psicolaborales y mejoren el clima laboral.

\section{REFERENCIAS BIBLIOGRÁFICAS}

1. Álvarez R. El síndrome de burnout: síntomas, causas y medidas de atención en la empresa. Éxito Empres. 2011;160.

2. Sánchez Alonso P, Sierra Ortega VM. Síndrome de Burnout en el personal de enfermería en UVI. Enferm Glob. 2014;13(1):252-66.

3. Maldonado E, Samudio M. Valoración De Las Subescalas De Maslach Burnout Inventory En Personal De Enfermería. Eureka. 2015;12(1):7-24.

4. Gutiérrez JJB, Arias-Herrera A. Síndrome de burnout en personal de enfermería: asociación con estresores del entorno hospitalario, Andalucía, España. Enfermería Univ. 2018;15(1).

5. Guillén RS. Síndrome de Burnout en profesionales de enfermería en el ámbito hospitalario: un estudio descriptivo. Rev Enfermería del Trab. 2017;7(3):65-9.

6. Olivares Faúndez V. Laudatio: Dra. Christina Maslach, Comprendiendo el burnout. Cienc y Trab. 2017;19(58):59-63.

7. Melita Rodríguez $A$, Cruz Pedreros M, Merino JM. Burnout en profesionales de enfermería que trabajan en centros asistenciales de la octava región, Chile. Cienc y Enferm. 2008;14(2):75-85.

8. Ecestaticos. Cuestionario Burnout - Maslach Burnout Inventory [Internet]. [citado 2020 Oct 1]. Disponible en: https://www.ecestaticos.com/file/0 35b15f97effea4c69fd6519e8114bcf/1515665476cuestionario_burnout.pdf

9. Muñoz SF, Ordoñez-Villota JN, Solarte-Moncayo $\mathrm{MN}$, Valverde-Ceron YC, Villarreal-Castro S, Zemanate-Perez ML. Síndrome de Burnout en enfermeros del Hospital Universitario San José. Popayán: Burnout syndrome in nurses from the University Hospital San José. Popayán. Rev Médica Risaralda. 2018;24(1):34-7. 


\section{ACERCA DE LOS AUTORES}

1. Eliana Marilin Cerón López: Enfermera profesional, Universidad Mariana, Colombia; Especialista en Perfusión en Cirugía Cardiovascular, Hospital Italiano de Buenos Aires, Buenos Aires, Argentina; Perfusionista, Hospital Clínica Guayaquil, Guayaquil, Ecuador.

ORCID: 0000-0002-4186-0248 